New design for temperature-strain discrimination using fiber Bragg gratings embedded in laminated composites

This article has been downloaded from IOPscience. Please scroll down to see the full text article. 2013 Smart Mater. Struct. 22105011

(http://iopscience.iop.org/0964-1726/22/10/105011)

View the table of contents for this issue, or go to the journal homepage for more

Download details:

IP Address: 193.144.200.57

The article was downloaded on 05/09/2013 at 08:27

Please note that terms and conditions apply. 


\title{
New design for temperature-strain discrimination using fiber Bragg gratings embedded in laminated composites
}

\author{
L Rodriguez-Cobo ${ }^{1}$, A T Marques ${ }^{2}$, J M López-Higuera ${ }^{1}$, J L Santos ${ }^{3}$ and \\ O Frazão ${ }^{3}$ \\ ${ }^{1}$ Photonics Engineering Group, Edificio I+D+i Telecom., University of Cantabria, E-39005 Santander, \\ Spain \\ 2 FEUP, Faculdade de Engenharia da Universidade do Porto, Rua Dr Roberto Frias, s/n, 4200-465 Porto, \\ Portugal \\ ${ }^{3}$ INESC Porto, Unidade de Optoelectrónica e Sistemas Electrónicos, Rua do Campo Alegre 687, \\ 4169-007 Porto, Portugal
}

E-mail: luis.rodriguez@unican.es

Received 4 April 2013, in final form 12 July 2013

Published 4 September 2013

Online at stacks.iop.org/SMS/22/105011

\begin{abstract}
A new smart structure based on fiber Bragg gratings (FBGs) embedded into composite laminates for temperature and strain simultaneous measurement has been designed and experimentally tested. Two holes have been drilled at preset locations in the composite plate to create different strain sensitivities at different locations. The proposed design has been compared to three reference sensing heads also based on embedding FBGs into composite materials. Experimental results agree remarkably well with mechanical simulations and validate all the tested designs for the temperature-strain discrimination. Based on the same principle, another sensing head with a long single FBG embedded has also been designed and experimentally tested, obtaining temperature independent strain measurement.
\end{abstract}

(Some figures may appear in colour only in the online journal)

\section{Introduction}

Composite materials technology has been widely applied to design and manufacture in a lot of new different scenarios. A wide set of applications from civil engineering to the aerospace industry has adapted this technology to overcome their limits. This growing technology is a perfect scenario in which to add sensing capabilities for studying their properties during material development. In particular, optical fiber sensors [1] have been proved as a highly compatible technology to be embedded into these composite materials, making it possible to obtain real time measurements during the manufacturing process and even under operation, when the final structure has been completed.

Within optical fiber sensors, fiber Bragg gratings have also been reported as very plausible sensing solutions to be embedded into composite materials [2]. They have been applied to monitor composite curing processes [3], to evaluate patch repairs of composite structures $[4,5]$ or even to detect composite damage [6], but one common problem is the thermal dependence of this kind of sensor. The measured reflection peak of an FBG depends both on deformation and on temperature, thus, when strain measurements have to be made in environments without temperature control, the employed FBG sensors require thermal compensation.

There are several works that deal with the simultaneous measurement of strain and temperature, such as different types of FBG [7], superstructure gratings [8], reversed index gratings [9] or even FBGs printed in special optical fibers such as bowtie [10] or microstructured [11]. However, most of the described solutions are unsuitable for structural health monitoring (SHM) purposes [12], where reliability and cost requirements are highly relevant. 

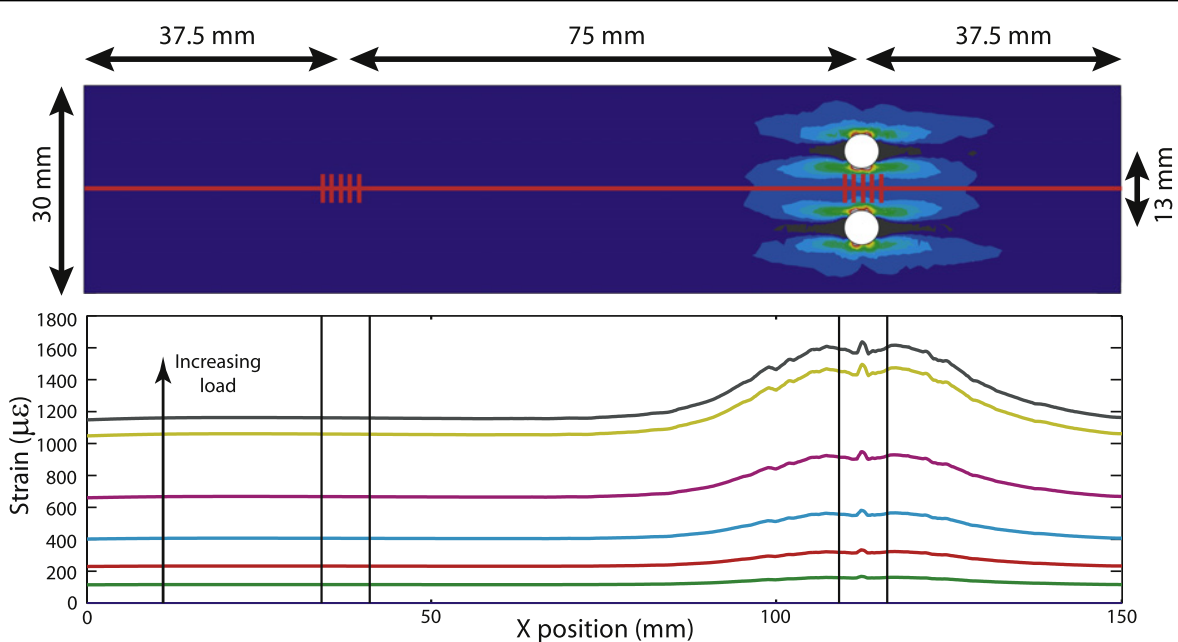

Figure 1. Simulated deformation of the holed design (top). Strain profile along the middle axis of the piece for different loads (bottom).

By embedding FBGs into composite materials, different solutions have also been reported to deal with the temperature compensation task. These reported solutions create different responses when strain and/or temperature are applied to a pair of embedded FBGs just by employing a different number of layers [13] or changing composite material within the FBG pair [14]. Despite reported solutions being more suitable for SHM purposes (based on a reliable and mature technology), their manufacture process can be simplified by employing the same host material.

In this work, a new sensor design based on drilling different holes at particular plate locations is compared to other sensing heads built in the same host material. Proposed designs are based on embedding FBGs into a constant thickness carbon fiber reinforced plastic (CFRP) plate whose shape has been designed to provoke different strain sensitivities along their middle axis. All the proposed designs have been simulated using the Abaqus finite element analysis (FEA) software (Dassault Systèmes) to study their mechanical response before its manufacture and experimental test. Optical spectra, simulated using the $T$-matrix method implemented over Matlab, agree remarkably with the loading test results. The achieved results also enable this new sensing head to perform temperature independent strain measurements just by employing a single long FBG.

\section{Strain-temperature sensor design}

The main goal of this work is to develop fiber Bragg grating (FBG) sensors based on CFRP plates capable of measuring both strain and temperature simultaneously. Designed sensors must be easy to manufacture, thus, maintaining a constant thickness, the shapes of CFRP plates are modified to provoke different strain responses to each FBG of an embedded pair. Since the hosting material is the same, the thermal sensitivities of the two FBGs must be similar, so, by increasing the strain sensitivity difference, a better strain-temperature discrimination must be obtained [15]. Based on a pair of embedded FBGs and employing four layers of unidirectional
CFRP, a new holed design has been compared to three reference plates both simulated and tested experimentally. Moreover, the new sensor design based on drilling the plate at preset locations has been evaluated to obtain temperature independent strain measurements using a single (but long) FBG.

\subsection{Holed design}

Starting from a rectangular plate of $150 \mathrm{~mm} \times 30 \mathrm{~mm}$, two holes of the same diameter are drilled in one side of the plate to increase its strain sensitivity. Holes are symmetrically located with respect to the middle axis of the plate, where the optical fiber is embedded. As drilling diameter is increased, strain sensitivity within the surrounding area also increases. For a given distance between holes, there is an optimum diameter that creates a practically flat deformation in the central area. However, strain sensitivity can still be increased by enlarging the drilling diameter, but the strain profile within the FBG length may not be so flat and may deform the FBG's spectral response.

In figure 1 , the simulated strain profile of a holed plate with two holes of $d=5 \mathrm{~mm}$ diameter separated by $D=$ $13 \mathrm{~mm}$ is depicted. For the chosen hole diameter $(d=$ $6 \mathrm{~mm}$ ), the best drilling separation must be $D \approx 13 \mathrm{~mm}$ to obtain a flatter deformation. If the drilling separation is lower, deformation in the middle axis is driven by the section reduction caused by the holes, thus the central area is more deformed than the side areas. This effect can be observed in figure 1 (bottom) with the appearance of a small peak within the central area. Otherwise, when the drilling separation is higher, strain in the central axis is driven by the combination of side effects due to stress redistribution of both holes, causing a lower strain in the central area than in the side areas.

When a flat section is required, there is a trade-off between hole diameter and center-to-center distance to obtain a central section deformation equal to side deformation (driven by stress redistribution). However, if a wide flat section is not required (i.e. by employing a short FBG), 


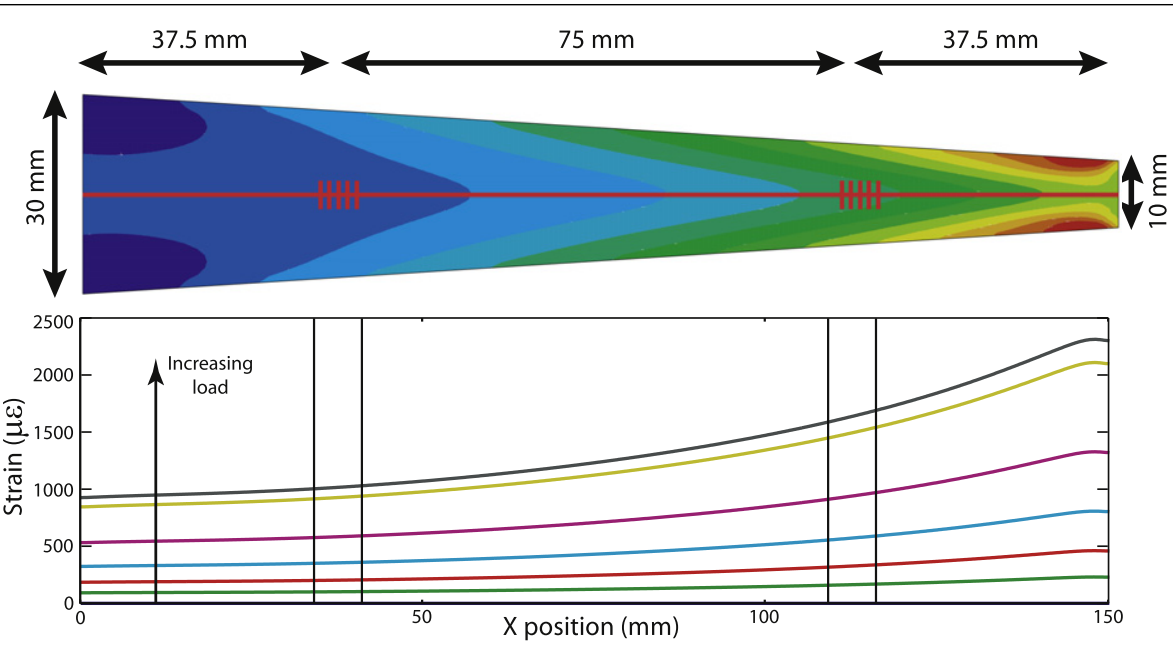

Figure 2. Simulated deformation of the triangular plate (top). Strain profile along the middle axis of the piece for different loads (bottom).

the hole diameter can be increased to achieve a greater deformation, but a deformation peak will appear at the central section. This effect can be avoided by placing the FBG slightly misaligned with respect to the holes' center axis, leaving the deformation peak out of the FBG.

\subsection{Triangular design}

For this design, the plate width is linearly decreased from one edge to the other: as the plate width decreases, its strain increases. Depending on the material properties, there is a maximum width gradient (width reduction slope) to take advantage of, because when the gradient is too high stress is not redistributed over the whole new width, so width variation is rendered useless. Maintaining the plate length of $150 \mathrm{~mm}$, a decreasing width variation from $d_{1}=30 \mathrm{~mm}$ to $d_{2}=10 \mathrm{~mm}$ has been simulated and is depicted below.

The simulated strain distribution of a triangular plate is depicted in figure 2. When the cross section is not uniform, applied stress has to be redistributed over the new available section, but, depending on material properties, stress redistribution cannot follow cross section variation and the stress is distributed over a smaller section. In the depicted situation, stress redistribution seems to work properly, being quicker at narrower sections and softer when cross section increases.

The achieved strain sensitivity difference between areas where FBGs are supposed to be is enough for the design purposes ( $\Delta \epsilon \approx 40 \%$ for the depicted plate), and this difference depends directly on the width difference (when stress redistribution works properly). However, the strain profile applied to an FBG placed in the narrow section (more deformed) is not flat, thus, when a high deformation is applied, the FBG spectrum may be deformed.

\subsection{Different widths}

The plate is divided into two sections of different widths to provoke a strain difference. Strain sensitivity variation is driven by two factors: the difference between widths and stress redistribution from the narrow section to the wider. A narrower section entails an increase in strain sensitivity; however, stress redistribution to a wider section may turn out to be less efficient. Depending on the chosen material, the trade-off between length and width has to be trimmed to enhance the strain sensitivity difference.

In figure $3, \mathrm{FEA}$ simulations of a $150 \mathrm{~mm}$ length plate that has a width reduction from $d_{1}=30 \mathrm{~mm}$ to $d_{2}=10 \mathrm{~mm}$ are depicted. Due to the constant width of the narrower section, the strain profile of this area becomes flat, making it possible to measure higher deformations without distorting the FBG spectrum. As this happens to the triangular plate, this design is also limited by the capability of the hosting material to redistribute the stress. However, since sections of the same width are larger, the achieved strain difference becomes greater ( $\Delta \epsilon \approx 50 \%$ for the simulated plate). The main drawback of this design is the transition section, where corners create weak points where stress becomes higher.

\subsection{Different widths (chamfered)}

The previous design has been improved to overcome the punctual stress locations due to corners. A chamfered section has been introduced to smooth the stress redistribution, decreasing punctual stresses. This design exhibits the same benefits as the previous one: a flatter strain distribution in the narrow section and a great strain sensitivity difference (above $\Delta \epsilon \geq 50 \%$ ). A width reduction from $d_{1}=30 \mathrm{~mm}$ to $d_{2}=10 \mathrm{~mm}$ has been performed over a total length of $L=150 \mathrm{~mm}$ using a chamfered section of $L_{\mathrm{c}}=10 \mathrm{~mm}$ length.

Achieved results of a chamfered plate are depicted in figure 4. It can be observed that the strain is spread better across the new section than in the previous design, achieving a greater strain sensitivity difference. Despite there still being some punctual stress concentrations due to corners, the absolute value is lower than in the non-chamfered design. This effect can be minimized by employing curved chamfers, but the sensitivity difference may also change. Some slight sensitivity improvement can be achieved by enlarging the 


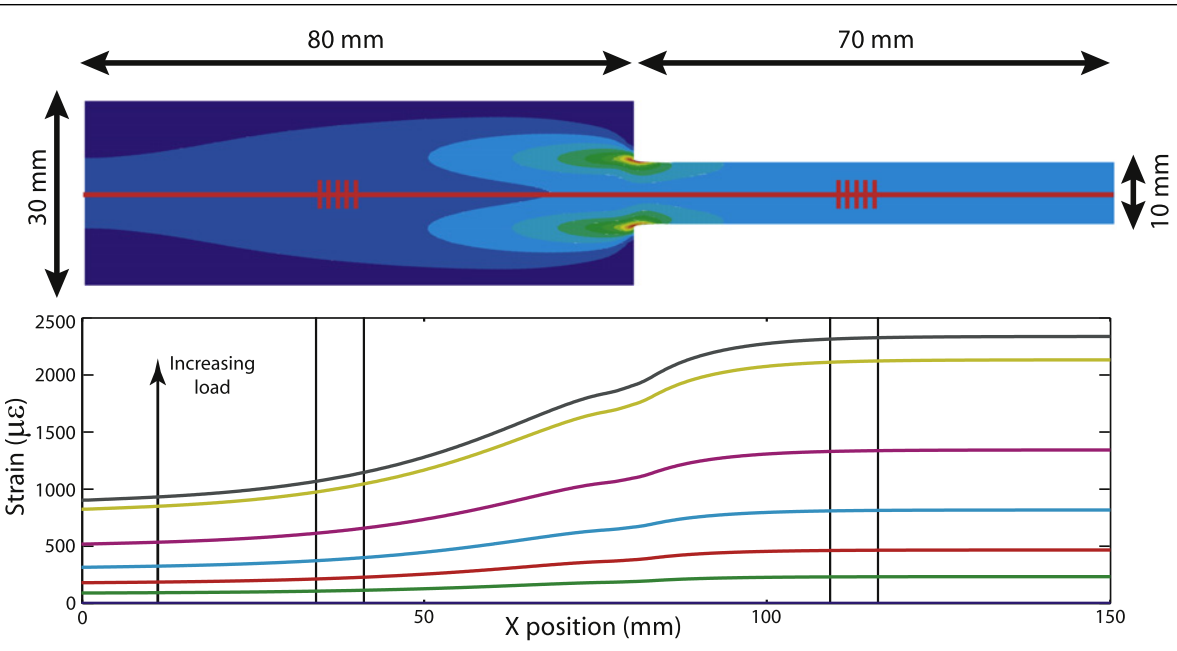

Figure 3. Simulated deformation of the plate with different widths (top). Strain profile along the middle axis of the piece for different loads (bottom).

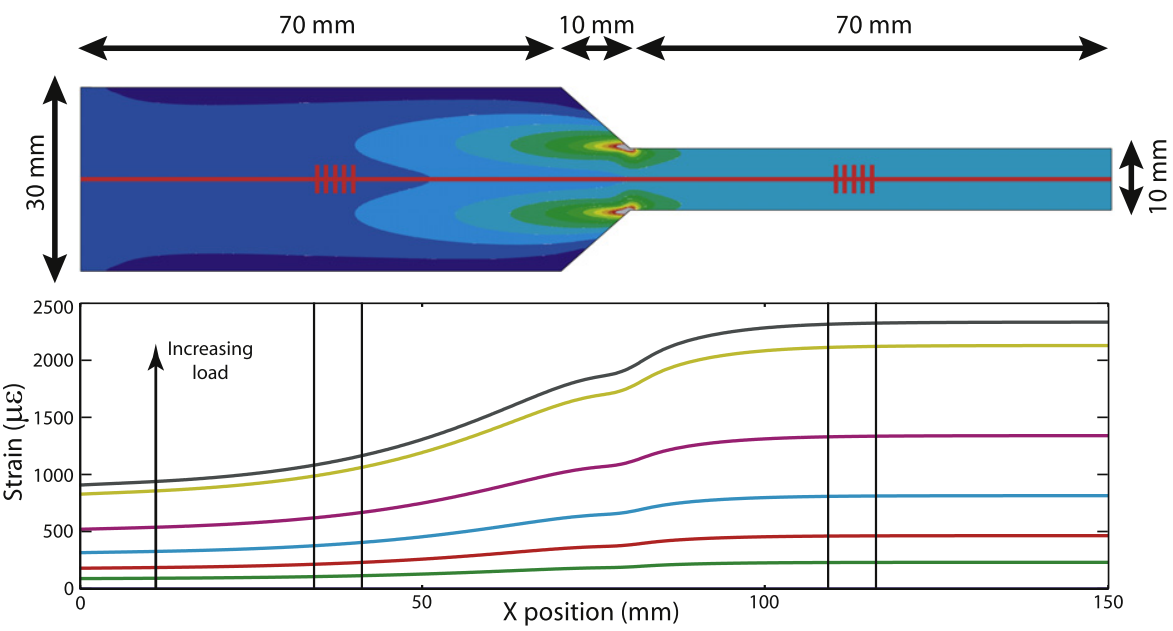

Figure 4. Simulated deformation of the chamfered plate (top). Strain profile along the middle axis of the piece for different loads (bottom).

chamfered section (i.e. with a length of $L_{\mathrm{c}}=20 \mathrm{~mm}$, the strain sensitivity difference should be $\Delta \epsilon \approx 53 \%$ ).

\section{Plate manufacture}

All the proposed designs have been manufactured using four layers of HS160REM prepreg using unidirectional orientation $(0-0-0-0)$. Optical fiber sensors have been embedded in the middle of the CFRP aligned to the middle axis of all plates. Employed fiber Bragg gratings were of $L=6 \mathrm{~mm}$ length with a reflectivity of $70 \%$ and have been written in standard optical fiber separated by $D_{\mathrm{FBG}}=75 \mathrm{~mm}$ using two different wavelengths.

After cutting four layers for each desired shape, two layers of each design are placed over the curing table before embedding the FBGs. Once aligned, the optical fiber is glued to the carbon prepreg far from the FBGs to maintain its position, having no influence on the strain distribution. Two polyimide tubes have been placed on both sides of the fiber to protect it at their exits from the carbon plate. Two remaining carbon layers were added before starting the curing process.
Once ready, the four plates were placed in an autoclave for $60 \mathrm{~min}$ at a temperature of $130^{\circ} \mathrm{C}$ with a pressure of $2 \mathrm{bar}$, the vacuum bag being at -0.5 bar.

All the plates have been enlarged by $\delta L=40 \mathrm{~mm}$ on both sides to clamp the plates at the loading machine, having a total length of $L_{\mathrm{T}}=230 \mathrm{~mm}$. All the plates have been manufactured with their final shape, with the exception of the holed plate (figure 5), in which, starting from a rectangular plate, two holes of $6 \mathrm{~mm}$ diameter have been drilled at preset locations. Strain and temperature responses of the resulting plates have been obtained and are described below.

\section{Strain and temperature responses}

Using a commercial FBG interrogating system (FiberSensing FS2200) with an accuracy of $2 \mathrm{pm}$, both strain and temperature characterizations have been performed for each manufactured plate. To obtain strain sensitivities, a loading test machine (Instron model 5869) has been employed to apply different loading cycles, keeping the plate temperature constant $\left(T^{\circ}=25^{\circ} \mathrm{C}\right)$. To obtain the thermal sensitivity, a 


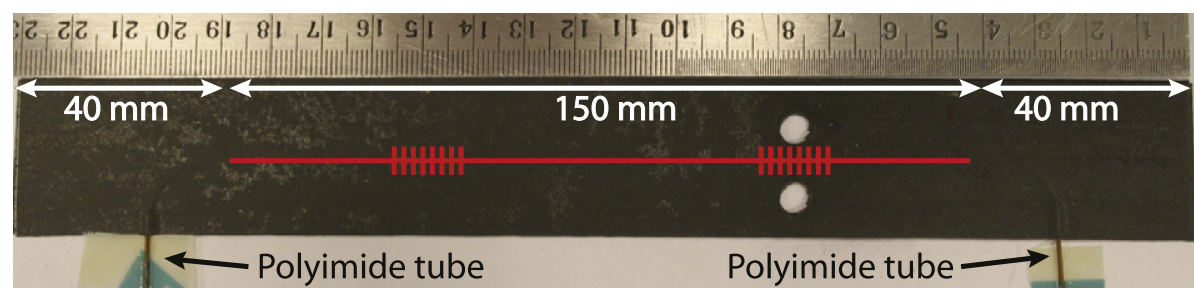

Figure 5. Detailed photo of the holed plate after drilling the holes. Two polyimide tubes have been added to the fiber to protect it from exiting the composite.
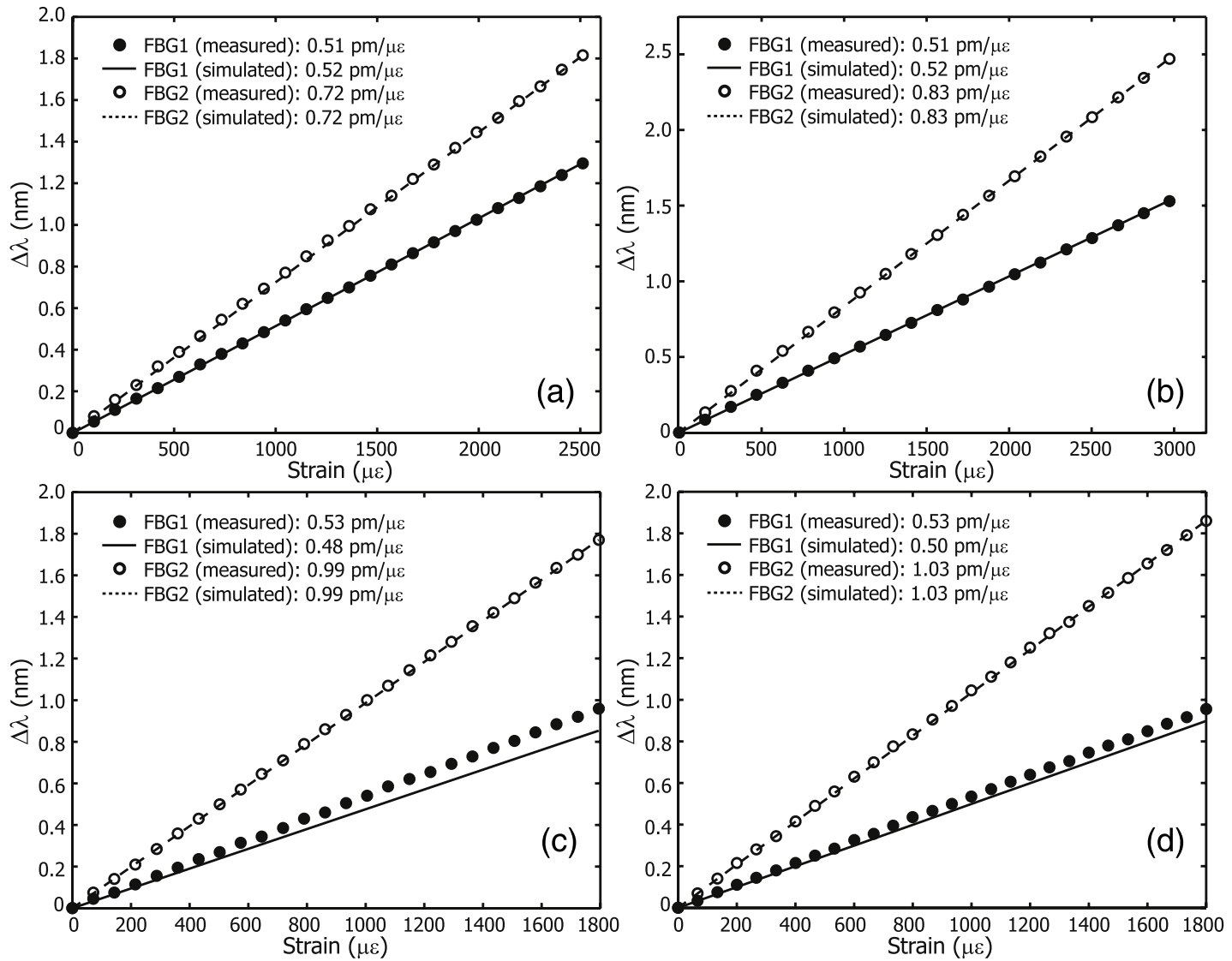

Figure 6. The achieved strain response of each plate is compared to simulated ones (dashed lines) of the holed plate (a), triangular (b), different widths (c) and chamfered (d).

temperature sweep from 30 to $70^{\circ} \mathrm{C}$ has been performed without applying any load to the plates.

\subsection{Strain response}

Five cycles reaching the same peak load have been performed for each plate. Initial cycles were performed to overcome the accommodation effect of FBG sensors to the host material [16]. The peak load has been trimmed for each plate (from 1 up to $3 \mathrm{kN}$ ), maintaining the maximum deformation under $\Delta s \leq 3000 \mu \epsilon$ and taking into account the available clamping area of each plate. All the applied cycles have a rising and falling time of $t=30 \mathrm{~s}$.

In figure 6 , the achieved strain response of each plate is compared to FEA simulations. All the achieved results agree remarkably well with their simulated responses, having a sensitivity around $\kappa_{\epsilon} \approx 0.5 \mathrm{pm} / \mu \epsilon$ for wider sections, that correspond to less sensitive FBGs. Due to the drilling process, a slight imbalance has been produced in the holed plate (both holes are displaced $0.5 \mathrm{~mm}$ off axis, but their relative separation has been maintained). This imbalance has been added to the FEA model to perform the simulation depicted in figure 6(a).

Due to the clamping system of the testing machine, a lower plate area has been clamped $(25 \mathrm{~mm}$ of the total width of $30 \mathrm{~mm}$ ), having no influence on the strain response of the holed and triangular designs, but it may introduce certain error in plates based on different widths. In addition to other factors, such as the possibility of acrylate coating slipping, designs based on different widths have some punctual stress concentration due to the width reduction, which may damage the plate under load. This damage is more critical for these 

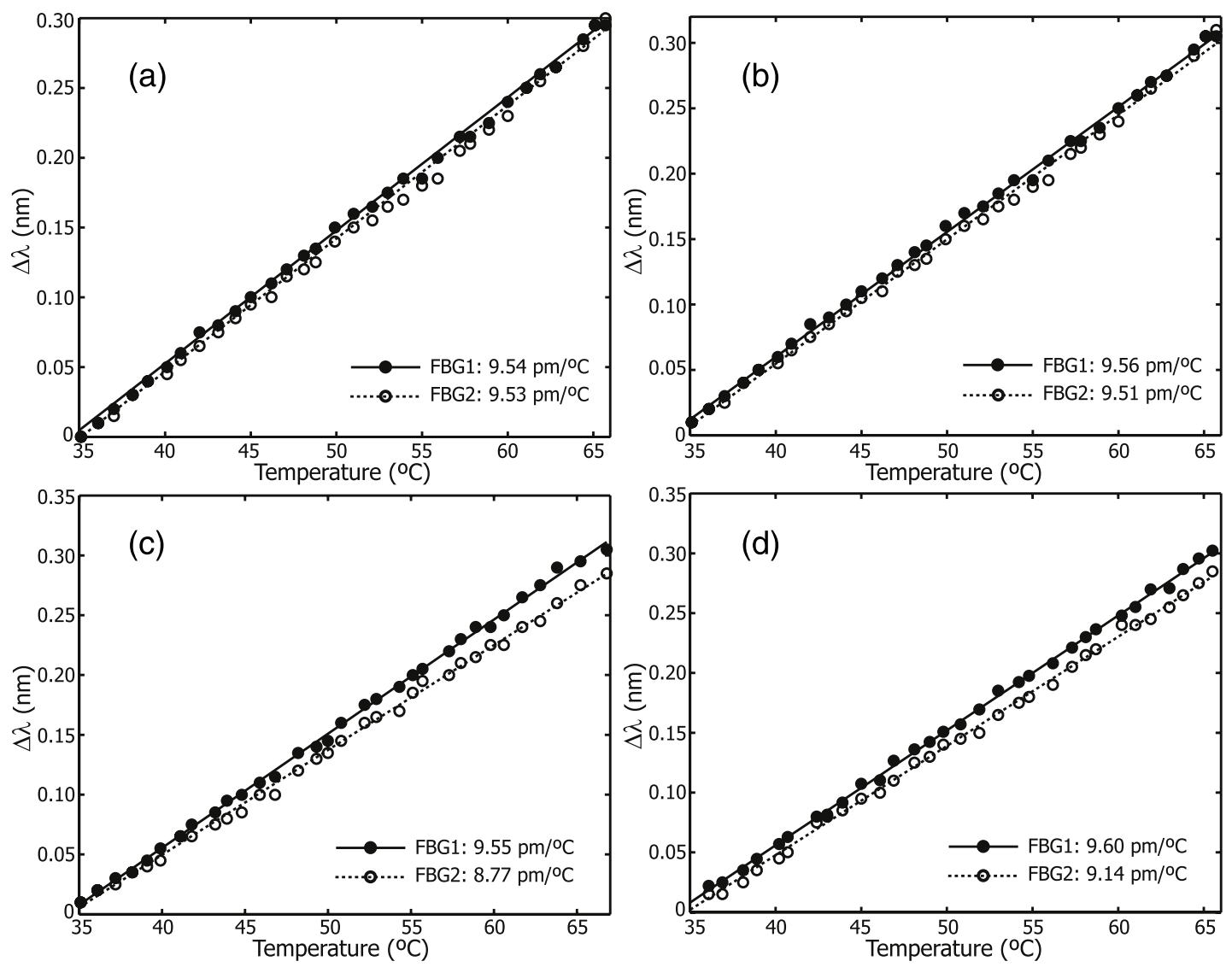

Figure 7. The achieved temperature response of each plate is depicted against its linear fit (dashed lines) for the holed plate (a), triangular (b), different widths (c) and chamfered (d).

designs, because strain redistribution is highly dependent on passing through high stress transition areas.

\subsection{Temperature response}

After obtaining the strain sensitivity of all plates, their thermal responses have been characterized by applying a temperature sweep $\left(35-70^{\circ} \mathrm{C}\right)$ to each plate without applying any load. During the sweep, spectra of both FBGs of each plate have been analyzed using the FiberSensing unit. Achieved wavelength drifts are depicted below.

The achieved thermal response is depicted in figure 7. Since all plates have been manufactured using the same host material, their thermal sensitivities are practically equal $\left(\kappa_{\mathrm{T}} \approx 9.5 \mathrm{pm}^{\circ} \mathrm{C}^{-1}\right)$. There are some slight differences due to the manufacture process such as FBG re-coating acrylate or slight fiber misalignment to the unidirectional (UD) carbon fiber. However, FBGs placed on narrower sections of plates of different widths (with higher strain sensitivity) exhibit a lower thermal sensitivity because surrounding CFRP has a lower epoxy resin ratio. This lack of resin is mainly caused by width reduction of the carbon plate, which it easier for resin to leak during the curing process (a re-coated FBG of $250 \mu \mathrm{m}$ is embedded into a $500 \mu \mathrm{m}$ thickness composite of $10 \mathrm{~mm}$ width).

\section{Results}

Since the proposed designs exhibit different sensitivities to strain, it is possible to obtain both measurands simultaneously from the wavelengths of each FBG pair of a plate [14]. Knowing the strain and temperature variations and having the sensitivities of both parameters, the Bragg wavelength of each FBG is given by

$$
\left[\begin{array}{l}
\Delta \lambda_{1} \\
\Delta \lambda_{2}
\end{array}\right]=\left[\begin{array}{ll}
\kappa_{\mathrm{T}_{1}} & \kappa_{\epsilon_{1}} \\
\kappa_{\mathrm{T}_{2}} & \kappa_{\epsilon_{2}}
\end{array}\right]\left[\begin{array}{l}
\Delta T \\
\Delta \epsilon
\end{array}\right]
$$

$\kappa_{\epsilon}$ and $\kappa_{\mathrm{T}}$ being the strain and temperature sensitivities of each FBG of a pair, thus, by obtaining the inverse matrix of the relationship, both measurands can be obtained from the Bragg wavelengths (equation (2)):

$$
\left[\begin{array}{c}
\Delta T \\
\Delta \epsilon
\end{array}\right]=\frac{1}{D}\left[\begin{array}{cc}
\kappa_{\epsilon_{2}} & -\kappa_{\epsilon_{1}} \\
-\kappa_{\mathrm{T}_{2}} & \kappa_{\mathrm{T}_{1}}
\end{array}\right]\left[\begin{array}{l}
\Delta \lambda_{1} \\
\Delta \lambda_{2}
\end{array}\right]
$$

where $D=\kappa_{\epsilon_{2}} \kappa_{\mathrm{T}_{1}}-\kappa_{\epsilon_{1}} \kappa_{\mathrm{T}_{2}}$ is the matrix determinant. The stability of this method can be evaluated using this determinant [15]: the higher its value, the more accurate are the results. Since the strain sensitivity of each FBG pair is different, the determinant of each FBG pair must be non-zero; however, achieved errors depend on the strain sensitivities' difference. 

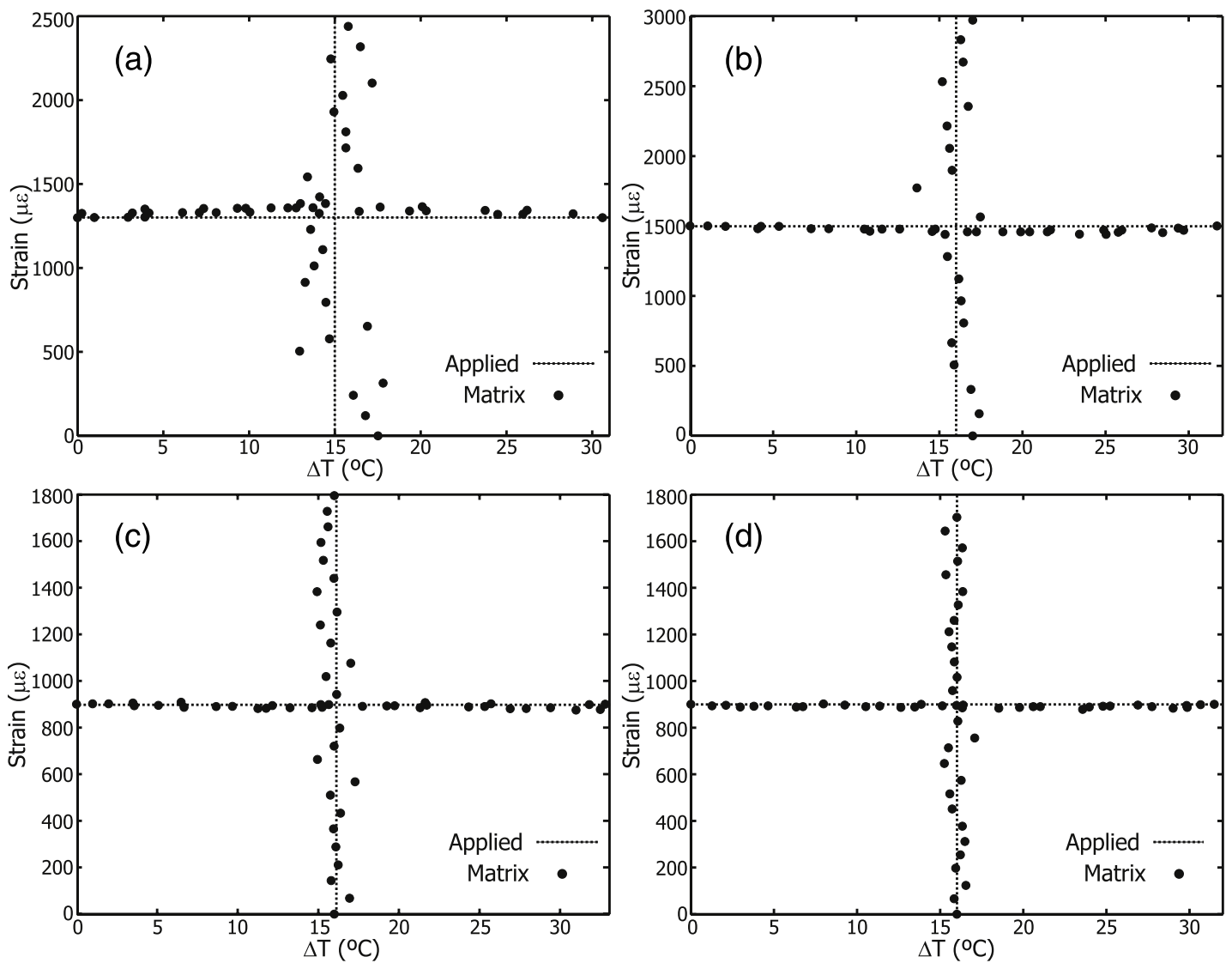

Figure 8. Resolution of the four designs: holed plate (a), triangular (b), different widths (c) and chamfered (d) under strain ramp at constant temperature and under a temperature sweep at constant load.

Table 1. Experimental sensitivities and errors of the tested designs.

\begin{tabular}{lccll}
\hline & Holed & Triangular & $\begin{array}{l}\text { Diff. } \\
\text { widths }\end{array}$ & Chamfered \\
\hline Strain $\mathrm{FBG}_{1}\left(\kappa_{\epsilon_{1}}\right)(\mathrm{pm} / \mu \epsilon)$ & 0.51 & 0.51 & 0.53 & 0.53 \\
Strain $\mathrm{FBG}_{2}\left(\kappa_{\epsilon_{2}}\right)(\mathrm{pm} / \mu \epsilon)$ & 0.72 & 0.83 & 0.99 & 1.03 \\
Temp. $\mathrm{FBG}_{1}\left(\kappa_{\mathrm{T}_{1}}\right)\left(\mathrm{pm}^{\circ} \mathrm{C}^{-1}\right)$ & 9.54 & 9.56 & 9.55 & 9.60 \\
Temp. $\mathrm{FBG}_{2}\left(\kappa_{\mathrm{T}_{2}}\right)\left(\mathrm{pm}^{\circ} \mathrm{C}^{-1}\right)$ & 9.53 & 9.51 & 8.77 & 9.14 \\
Mean strain error $(\mu \epsilon)$ & 38.0 & 26.5 & 9.3 & 8.0 \\
Mean temp. error $\left({ }^{\circ} \mathrm{C}\right)$ & 2.19 & 0.97 & 0.49 & 0.32 \\
\hline
\end{tabular}

In figure 8 , the achieved resolution of all the plates is depicted. Each graph is obtained by varying one measurand while the other is fixed. For each plate, applied sweeps (strain and temperature) are depicted (solid lines) against the computed variations using wavelength drifts (dots). As the strain sensitivity of each FBG pair increases, computed results improve. A summarizing table of the obtained parameters is given in table 1.

All tested plates have been designed to be easy to manufacture, so, based on the same composite material (CFRP) and using the same thickness $(0.5 \mathrm{~mm})$, their twodimensional shapes have been modified to achieve different strain sensitivities on their embedded FBGs. Achieved results exhibit the capability to obtain both measurands (strain and temperature) using the wavelength drift of each FBG pair; however, the measured error of the tested plates is different.
Best results have been obtained employing two sections of different widths where both FBGs have been embedded, but this approach requires the narrowing of one side of the sensing head, making its installation more difficult. The holed design has a lower sensitivity difference to strain (and a higher error); however, the structure is symmetrical, which makes its installation process easier. Besides, this design can be improved by enlarging the holes and displacing them from the FBG center (avoiding the middle strain peak).

All the proposed designs are based on a pair of embedded FBGs to obtain both measurands (strain and temperature) simultaneously. However, just obtaining one measurand without having cross-sensitivity error (i.e. thermal error for FBGs) may be enough. Based on the holed design, another approach using a longer FBG has also been tested to obtain strain measurements without temperature dependence 


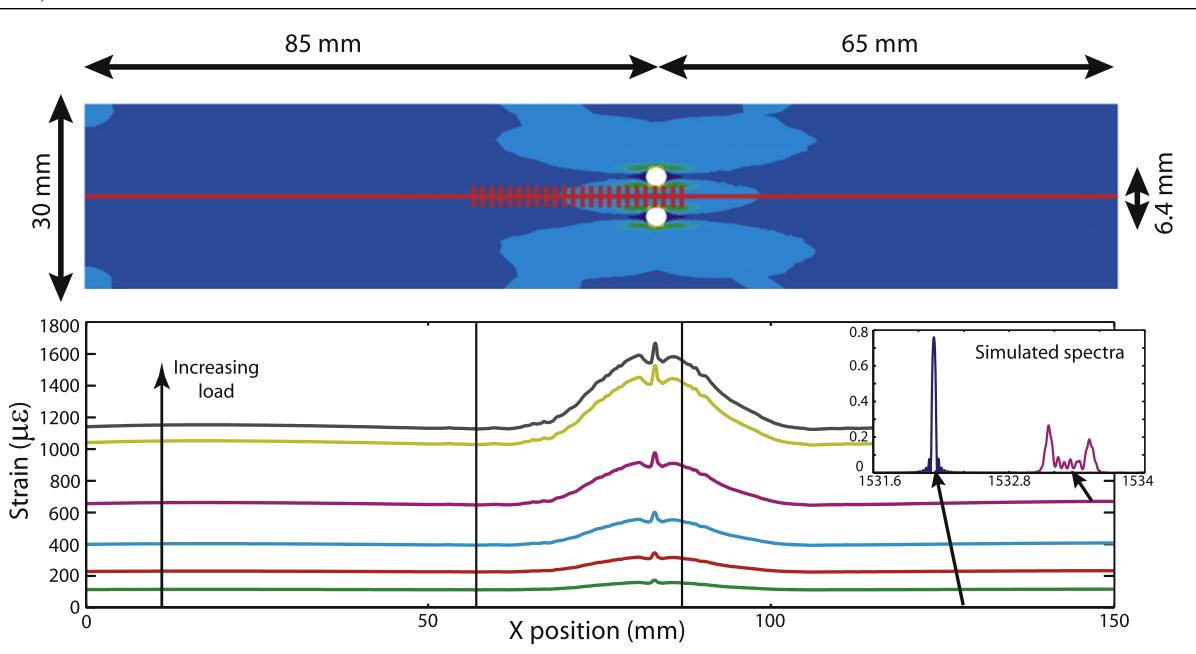

Figure 9. Simulated deformation of the temperature independent strain sensor (top). Strain profile along the middle axis of the piece for different loads (bottom) and their simulated FBG spectra.

by forcing two different strain sensitivities within the FBG length.

\section{Temperature independent strain sensor}

A single FBG of $L=30 \mathrm{~mm}$ is embedded in the middle of a rectangular CFRP plate of constant thickness $(0.5 \mathrm{~mm})$. To obtain temperature independent strain measurements, the main goal is to create two areas within the FBG length whose strain sensitivities were different. Steeper deformation profiles can be achieved by drilling holes closer to the fiber, so two small holes are placed close to one edge of the embedded FBG, creating two flat deformation areas: one close to the hole and the other on the opposite FBG edge. This strain sensitivity difference may split the single FBG spectral peak into two. The distance between these two peaks may be proportional to applied deformation but not to applied temperature.

\subsection{Design and simulation}

Based on a rectangular CFRP plate of $150 \mathrm{~mm} \times 30 \mathrm{~mm}$ of constant thickness, two small holes (of $D=3 \mathrm{~mm}$ ) are made close to one FBG edge. The area between holes exhibits a higher strain sensitivity, and, if the distance between holes and the central axis is related to their diameter, the achieved deformation profile must be flat. Since holes are closer to the fiber axis, the gradient of the strain sensitivity is greater, thus the strain profile goes quickly from a higher to a lower sensitivity area. This steeper deformation profile allows the creation of two flat deformation areas at both FBG edges.

In figure 9, FEA simulations of the proposed design are depicted. Two holes of $D=3 \mathrm{~mm}$ diameter separated by $d=6.4 \mathrm{~mm}$ and at $\mathrm{d} L=5 \mathrm{~mm}$ from one FBG edge have been simulated. The strain profile applied to the FBG consists of two flat areas separated by a transition area where the strain varies quickly. The two flat areas will split the FBG spectrum into two peaks, each one proportional to each flat deformation area.

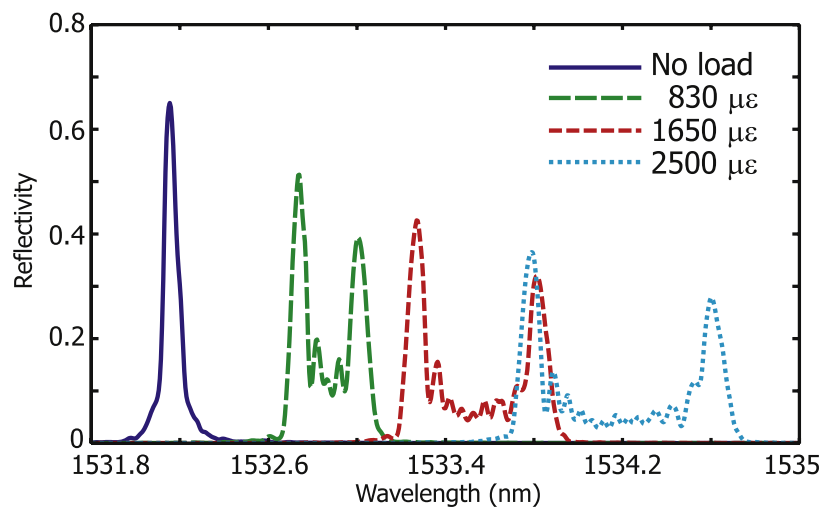

Figure 10. Measured FBG spectra of the manufactured plate under four different loads. Peak separation is proportional to the applied load.

\subsection{Experimental response}

After manufacturing the plate using the same procedure as in previous ones, two holes of $3 \mathrm{~mm}$ diameter have been drilled into the CFRP plate following the proposed design (depicted in figure 9). Once drilled, the plate has been clamped to the testing machine (Instron model 5869) to perform five loading cycles (with a rising and falling time of $t=30 \mathrm{~s}$ ) reaching a peak load of $3 \mathrm{kN}$. During the loading test, the FBG has been interrogated using a commercial system (FiberSensing FS2200), recording FBG spectra. In figure 10, measured FBG spectra for four different loads are depicted.

When no load is applied to the plate, the FBG spectrum keeps its original shape (single peak), but, when load is increased, the single FBG peak splits into two, each one corresponding to both flat deformation areas. The lower peak is more defined because its corresponding area (far from holes) is flatter than the area close to the holes (that provokes the other peak). The distance between the two spectral peaks is proportional to the applied strain. This structure can be understood as two FBGs with different strain sensitivities that 

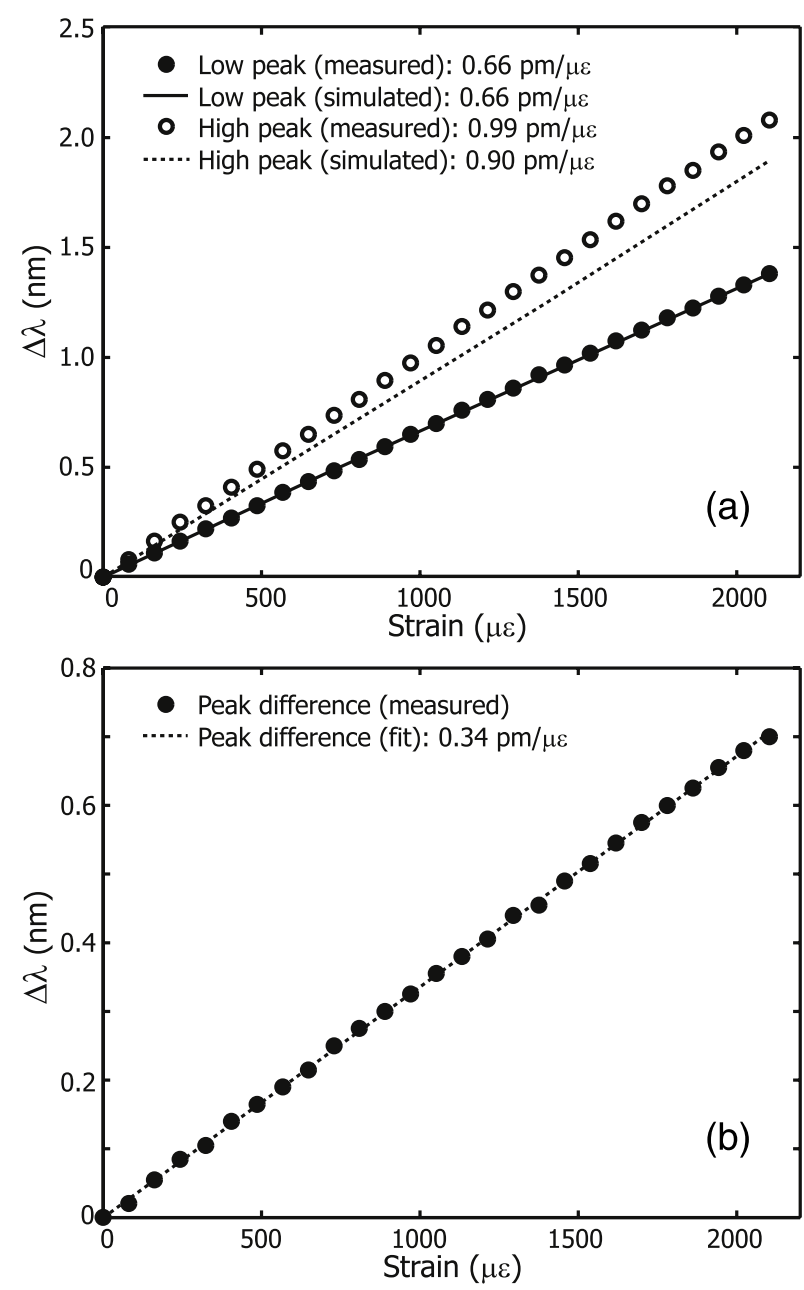

Figure 11. Measured (dots) and simulated (line) peak wavelength drift (a) and their relative separation (b).

share the same Bragg wavelength, thus their peak separation is proportional to their strain sensitivity difference.

From simulated deformation profiles, FBG spectra are computed using the $T$-matrix method for each different load. The peak separations of both measured and simulated spectra are detected and their separations are compared in figure 11. Simulated results have been scaled to the lower peak sensitivity. Experimental results exhibit a higher strain difference than does the simulated model $\left(\Delta \kappa_{\epsilon_{\mathrm{m}}} \approx\right.$ $0.34 \mathrm{pm} / \mu \epsilon$ against $\left.\Delta \kappa_{\epsilon_{\mathrm{s}}} \approx 0.24 \mathrm{pm} / \mu \epsilon\right)$; however, the expected peak splitting is reproduced. This plate has been manufactured using a non-re-coated FBG, so surrounding carbon sheets are closer to the optical fiber, having a smaller gap to be filled with epoxy resin. A lower epoxy resin concentration surrounding the optical fiber allows a better deformation transference from the carbon sheets, thus steeper deformation profiles are achieved.

After performing strain tests, the plate is subjected to a temperature sweep $\left(35-70^{\circ} \mathrm{C}\right)$ to obtain its thermal sensitivity. As shown in figure 7, plates manufactured with a homogeneous shape, where the mechanizing process has been performed after curing the composite (i.e. holed design), have a uniform thermal sensitivity (the ratio between carbon fiber
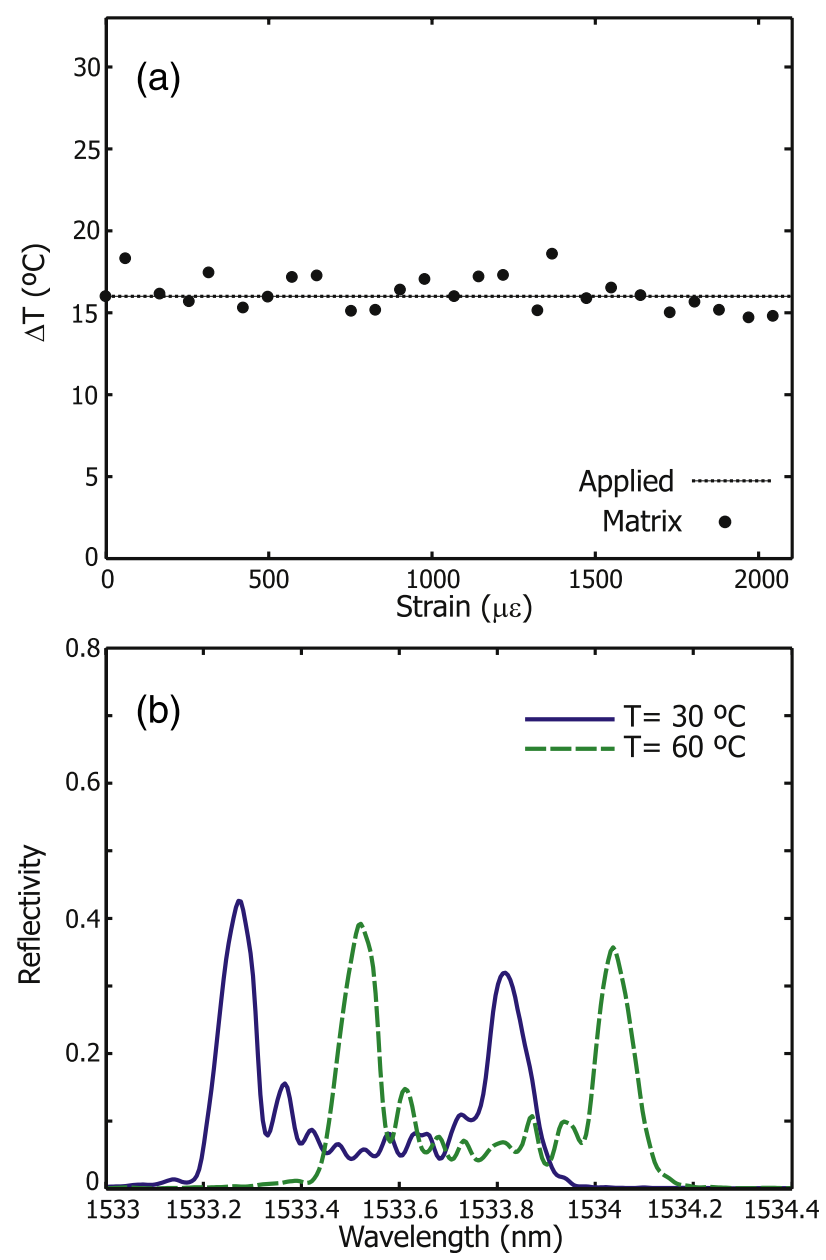

Figure 12. (a) Calculated values (dots) are compared to the temperature during the strain sweep. (b) Two FBG spectra under same load $(\Delta s \approx 1600 \mu \epsilon)$ but at different temperatures.

and epoxy resin is maintained). The achieved temperature sensitivity $\left(\kappa_{\mathrm{T}}=8.84 \mathrm{pm}^{\circ} \mathrm{C}^{-1}\right)$ seems to be lower than that obtained with re-coated FBGs $\left(\kappa_{\mathrm{T}_{\mathrm{R}}} \approx 9.50 \mathrm{pm}^{\circ} \mathrm{C}^{-1}\right)$, which suggests a lower epoxy resin concentration surrounding the optical fiber.

Since both peaks have the same thermal sensitivity $\left(\kappa_{\mathrm{T}}=8.84 \mathrm{pm}^{\circ} \mathrm{C}^{-1}\right)$ and the strain is proportional to their separation $\left(\Delta \kappa_{\epsilon \mathrm{P}} \approx 0.34 \mathrm{pm} / \mu \epsilon\right)$; the matrix equation detailed in equation (2) (considering each peak as an independent FBG) can be simplified for the strain value as

$$
\begin{aligned}
\Delta \epsilon & =\frac{1}{\kappa_{\mathrm{T}} \cdot\left(\kappa_{\epsilon_{\mathrm{P} 2}}-\kappa_{\epsilon \mathrm{P} 1}\right)} \cdot \kappa_{\mathrm{T}} \cdot\left[\Delta \lambda_{\mathrm{P} 2}-\Delta \lambda_{\mathrm{P} 1}\right] \\
& =\frac{1}{\Delta \kappa_{\epsilon \mathrm{P}}} \cdot \Delta \lambda_{\mathrm{P}}
\end{aligned}
$$

where $\Delta \lambda_{\mathrm{P}}$ is the peak separation and $\Delta \kappa_{\epsilon \mathrm{P}}$ the difference of strain sensitivities.

In figure 12(a), temperature values calculated using the matrix equation are compared to the applied temperature while a deformation sweep is performed. The mean temperature error is $\overline{\Delta T} \approx 0.81{ }^{\circ} \mathrm{C}$, with a maximum drift of $\Delta T \approx 2.60^{\circ} \mathrm{C}$ under a strain sweep of up to $\Delta s \approx 2200 \mu \epsilon$. 
In figure 12(b) two measured FBG spectra corresponding to two different temperatures $\left(T_{1}=30^{\circ} \mathrm{C}\right.$ and $\left.T_{2}=60^{\circ} \mathrm{C}\right)$ under the same loading condition $(\Delta s \approx 1600 \mu \epsilon)$ are depicted. The peak separation is proportional to the applied deformation, but their offset position is modified by the temperature so just temperature values are affected by the error, having no error on the strain discrimination.

\section{Discussion}

All the proposed designs agree remarkably well with the simulated models. Despite the holed design having a lower strain sensitivity difference than the other tested plates, it has some advantages in temperature response. All tested designs are based on the same manufacturing process and the same material and thickness, but there are still some differences that make the holed design able to obtain temperature independent strain measurements.

In order to simplify the manufacturing process, reference designs (triangular and different widths) have been cut before curing. In contrast, on holed plates, it is easier to drill the holes after curing. Despite the curing process having been performed under the same pressure and temperature conditions, the resulting amount of epoxy resin surrounding the optical fiber was not the same. For designs where a narrower section is required (i.e. different widths), resin leak is more relevant due to the chosen dimension and materials, leaving less resin surrounding the optical fiber. This situation is noticed by a lower thermal sensitivity at narrower sections and it complicates temperature independent strain measurements. On the other hand, holes have been drilled after the curing process so the surrounding epoxy resin was maintained, achieving the same thermal sensitivity along the plate. Despite the achieved difference of strain sensitivity of the holed plate being lower than reference designs, its uniform thermal sensitivity partially compensates the lack of strain sensitivity, achieving a relatively good strain discrimination; however, a higher difference between temperature sensitivities is required to improve temperature discrimination.

Besides, uniform temperature sensitivity makes it possible to make temperature independent strain measurements by embedding a single FBG into a carbon plate. Two small holes are drilled in the plate to force the FBG spectrum to split into two peaks, whose difference is proportional to deformation. This configuration exhibited an error-free strain discrimination without temperature cross-sensitivity.

Based on easy-to-manufacture configurations, several composite plate designs have been manufactured and tested, exhibiting good results on strain-temperature discrimination. All the proposed designs have been manufactured using CFRP but, since strain sensitivity difference is determined by plate shape, other host materials can be employed to obtain sensing heads more suitable for the desired application. The proposed designs can be installed by gluing both edges (clamping areas) or can also be considered in the structure design to be included as structural parts, performing both tasks, mechanical reinforcement and sensing areas.

\section{Conclusion}

In summary, a new sensing head for strain-temperature discrimination based on embedding fiber Bragg gratings into composite plates has been designed and experimentally tested. The design relies on drilling two symmetrical holes to provoke different strain sensitivities in embedded FBGs, and it has been compared to three reference composite designs. The achieved results agree remarkably well with the simulated response, making it possible to obtain both measurands (strain and temperature) simultaneously. Besides, due to the uniform temperature sensitivity of the proposed design, a temperature independent strain sensor has been also manufactured and tested. By creating a non-uniform strain profile on an embedded long FBG, its spectral peak has been split into two, its separation being proportional to plate deformation. Since the characteristics of the proposed design are given by its shape, the employed hosting material can be changed to fit the desired application.

\section{Acknowledgments}

Special thanks to FiberSensing, Sistemas Avançados de Monitorização, for its support and valuable collaboration with the experimental works. This work has been supported by ICT COST Action TD1001 (OFSeSa) and the Spanish grant AP2009-1403 and TEC2010-20224-C02-02.

\section{References}

[1] López-Higuera J M 2002 Handbook of Optical Fiber Sensing Technology (New York: Wiley)

[2] Kuang K, Kenny R, Whelan M, Cantwell W and Chalker P 2001 Compos. Sci. Technol. 61 1379-87

[3] Murukeshan V, Chan P, Ong L and Seah L 2000 Sensors Actuators A 79 153-61

[4] McKenzie I, Jones R, Marshall I and Galea S 2000 Compos. Struct. 50 405-16

[5] Takeda S, Yamamoto T, Okabe Y and Takeda N 2007 Smart Mater. Struct. 16763

[6] Propst A, Peters K, Zikry M, Schultz S, Kunzler W, Zhu Z, Wirthlin M and Selfridge R 2010 Smart Mater. Struct. 19015016

[7] Shu X, Liu Y, Zhao D, Gwandu B, Floreani F, Zhang L and Bennion I 2002 Opt. Lett. 27 701-3

[8] Guan B O, Tam H Y, Tao X M and Dong X Y 2000 IEEE Photon. Technol. Lett. 12 675-7

[9] Park S, Jang B, Lee Y, Kim C and Park C 2010 Meas. Sci. Technol. 21035703

[10] Urbanczyk W, Chmielewska E and Bock W J 2001 Meas. Sci. Technol. 12800

[11] Frazão O, Carvalho J, Ferreira L, Araújo F and Santos J 2005 Meas. Sci. Technol. 162109

[12] López-Higuera J M, Rodriguez Cobo L, Quintela Incera A and Cobo A 2011 J. Lightwave Technol. 29 587-608

[13] Frazão O, Oliveira R and Dias I 2009 Microw. Opt. Technol. Lett. $51235-9$

[14] Ferreira M, Vieira J, Frias C and Frazão O 2011 Meas. Sci. Technol. 22045206

[15] Jin W, Michie W C, Thursby G, Konstantaki M and Culshaw B 1997 Opt. Eng. 36 598-609

[16] Pereira G, Frias C, Faria H, Frazão O and Marques A 2012 Polym. Test. 32 99-105 